\section{RELATION BETWEEN N-TERMINAL PRO B-TYPE NATRIURETIC PEPTIDE (NT-PROBNP) AND DISEASE SEVERITY IN PAEDIATRIC HYPERTROPHIC CARDIOMYOPATHY}

'Laxmi Kaliyappan, 'Sarah Watson, ${ }^{2}$ Ella Fiend, ${ }^{1,2}$ Gabrielle Norrish, ${ }^{2}$ Elena Cervi, 1,2Juan Kaski. 'Institute of Cardiovascular Science, UCL, Fitzrovia, UK; ${ }^{2}$ Centre for Inherited Cardiovascular Diseases, Great Ormond Street Hospital, London UK

\subsection{6/heartjnl-2021-BCS.7}

Introduction N-terminal pro B-type natriuretic peptide (NTproBNP) is associated with an increased risk of mortality and heart failure related adverse events in adults with hypertrophic cardiomyopathy (HCM). Elevated NT-proBNP levels have been correlated with multiple subjective and objective parameters of HCM severity including dyspnoea and left ventricular maximal wall thickness (LVMWT). However, robust prognostic markers in adults may not be reliable for children with HCM in whom disease severity assessment is challenging. No studies have yet evaluated utility of NT-proBNP in children with HCM.

Thus, the objective of this study was to assess associations of NT-proBNP with conventional markers of disease severity and predictive ability of NT-proBNP in a paediatric HCM cohort.

Methods Plasma NT-proBNP levels were measured in eighty consecutive patients [23 (28.8\%) females; median age: 12.3 years (interquartile range (IQR): 6.4-16.0); 37 (46.3\%) sarcomeric aetiology]. Contemporaneous data from conventional clinical evaluation was used to establish disease severity including electrocardiography, echocardiography, tissue Doppler imaging, magnetic resonance imaging (MRI) and cardiopulmonary exercise testing.

Results Median NT-proBNP concentration was $1104.5 \mathrm{pg} / \mathrm{mL}$ (range: 20-11206 pg/mL and IQR: 108.5-2613.5 pg/mL). NT-proBNP levels correlated with: QTc $(\rho=0.445, \mathrm{p}<0.01)$; septal thickness z-score $(\rho=0.618, \quad \mathrm{p}<0.001)$; MLVWT zscore $(\rho=0.582, \mathrm{p}<0.001)$; lateral S' $(\rho=-0.668, \mathrm{p}<0.001)$; septal E/E' $(\rho=0.609, \quad \mathrm{p}<0.001) ;$ MRI MWT $(\rho=0.773$, $\mathrm{p}<0.001$; indexed LV mass $(\rho=0.576, \mathrm{p}<0.001)$ and peak systolic blood pressure $(\rho=-0.605, \mathrm{p}<0.001)$. There were

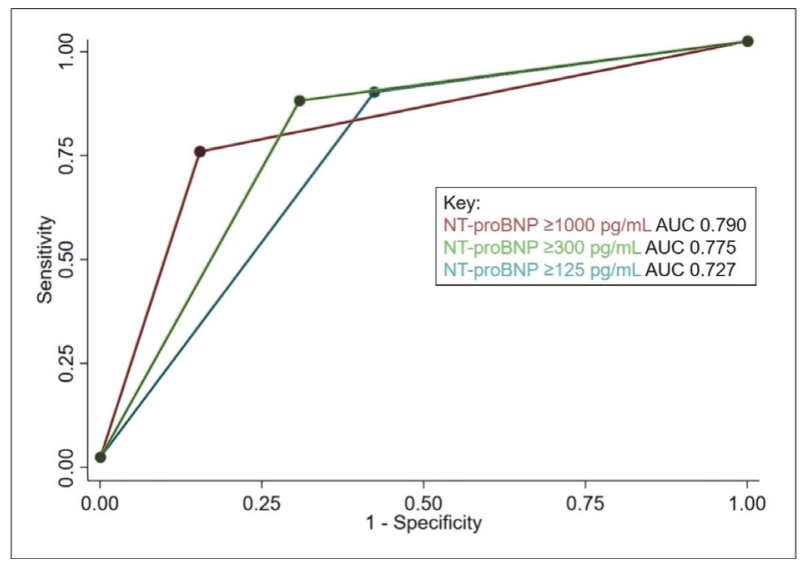

Abstract 7 Figure 1 Receiver operator characteristic (ROC) curves of NT-proBNP cut-offs for predicting septal E/E' $>10$, a marker of diastolic dysfunction, in children with hypertrophic cardiomyopathy. True-positive rate (sensitivity), false positive rate (1-specificity) and area under the curve (AUC) are displayed for the following cut-offs; NT-proBNP $\geq 1000$ $\mathrm{pg} / \mathrm{ml}$ (red), NT-proBNP $\geq 300 \mathrm{pg} / \mathrm{ml}$ (green) and NT-proBNP $\geq 125 \mathrm{pg} /$ $\mathrm{ml}$ (blue) weak associations between NT-proBNP and aetiology or subjective symptoms including palpitations and chest pain $(\mathrm{p}>0.05)$.

NT-proBNP levels were higher in patients who were: female; dyspnoeic (defined as Ross/NYHA Class $\geq \mathrm{II}$ ); prescribed cardioactive medication and had an implantable cardioverter defibrillator $(\mathrm{p}<0.05)$.Lateral $S^{\prime} \quad(\beta=-0.306$, $\mathrm{p}=0.001)$ and MLVWT $(\beta=0.217, \mathrm{p}=0.013)$ were independent predictors of NT-proBNP in multivariate analysis. At a cut-off point of $300 \mathrm{pg} / \mathrm{ml}$, NT-proBNP had a positive predictive value of $84 \%$ and a negative predictive value of $72 \%$ for predicting septal E/E'>10 (Area under the curve $=0.775$ $(\mathrm{p}<0.001))$ (See figure 1).

Conclusions NT-proBNP levels correlate with parameters of disease severity in paediatric HCM including measures of diastolic dysfunction (septal E/E') and systolic dysfunction (lateral S'). NT-proBNP measurement may be an effective adjunct for monitoring disease severity in children, particularly when conventional clinical evaluation is challenging. Future studies in larger cohorts of children are needed to explore prognostic value.

Conflict of Interest None

\section{SINGLE-CELL RNA SEQUENCING REVEALS CARDIAC CELL-SPECIFIC TRANSCRIPTOMIC CHANGES IN DILATED CARDIOMYOPATHY}

Oisín Cappa. Queen's University Belfast, Belfast, UK

\subsection{6/heartjnl-2021-BCS.8}

Introduction Dilated cardiomyopathy (DCM) is the most common cause of heart failure (HF), with a complex aetiology including lifestyle and genetic factors involving pathological changes in multiple cardiac cell types. The ability of single-cell RNA sequencing (scRNA-Seq) to measure gene expression in thousands of individual cells simultaneously provides a way to study the differing pathological changes in cell types within complex tissues. We aimed to detect celltype-specific transcriptomic alterations implicated in DCM through an integrated analysis of publicly available adult heart scRNA-Seq datasets that leveraged recent advancements in single-cell analytical tools.

Methods scRNA-Seq data from an adult human HF dataset containing $\operatorname{DCM}(n=5)$ and control $(n=14)$ samples were retrieved from Gene Expression Omnibus (GSE109816, GSE121893) and subjected to an updated bioinformatic workflow. Unsupervised clustering analysis of 10,242 cells was paired with reference celltype mapping from Heart Cell Atlas data to produce a more comprehensive annotation of the HF dataset. Differential expression analysis was performed between DCM and control cells to identify celltype-specific transcriptomic changes in DCM. Bulk RNA-seq was performed on adult human DCM $(n=9)$ and control $(n=9)$ heart tissue to detect whole-tissue changes. Genes differentially expressed in bulk and single-cell data were intersected to generate a list of putative DCM-linked genes, validated in vitro by RT-qPCR in human cardiac fibroblasts.

Results Our single-cell workflow resolved 8 distinct cell populations in the heart, 4 of which were not reported in the original publication associated with the data. The validity of these cell populations was strongly supported by the similarity of their transcriptomic profiles with those of the recently 
published Heart Cell Atlas study. Expression was altered in DCM in all but the rarest heart cell populations. Genes identified as altered in DCM in bulk RNA-seq were compared with altered genes from each single-cell cardiac cell population. Greatest concordance between the two techniques was noted in fibroblasts, with 14 upregulated and 13 downregulated genes common across both analyses. Several of these genes were independently validated in an in vitro model of TGF $\beta$-treated human cardiac fibroblasts.

Conclusions DCM is a complex pathology involving interactions between multiple cardiac cell populations. Our analysis workflow improved resolution at the single-cell level, providing more accurate recapitulation of in vivo tissue heterogeneity. This unbiased approach has enabled the robust detection of unique disease-relevant transcriptomic alterations in specific cardiac cell populations in DCM.

Conflict of Interest None

\section{INDUCED PLURIPOTENT STEM CELL-DERIVED ENDOTHELIAL CELLS FROM HUMAN DIABETIC DONORS CARRY AN IMPRINT OF THE DIABETIC MILIEU}

\begin{abstract}
${ }^{1}$ Magdalini Eleftheriadou, ${ }^{1}$ Hojjat Naderi Meskin, 'Andrew Yacoub, 'Thomas Morrison, ${ }^{1}$ Victoria Cornelius, ${ }^{2}$ Chunbo Yang, ${ }^{3}$ Martha Gonzalez, ${ }^{1}$ Sophia Kelaini, ${ }^{1}$ Garret Carney, ${ }^{1}$ Guillermo Lopez Campos, 'Noemi Lois, ${ }^{1}$ David Grieve, ${ }^{1}$ Alan Stitt, 'Andriana Margariti. ${ }^{1}$ Queens University Belfast, Belfast, UK; ${ }^{2}$ Newcastle University; ${ }^{3}$ Cambridge University
\end{abstract}

10.1136/heartjnl-2021-BCS.9

Diabetic endotheliopathy is the main cause for impaired angiogenesis and reduced neovascularization that lead to microvascular injury and vascular complications. The pathogenic basis for vascular complications arising from diabetes is complex. Elucidation of key underlying mechanisms will help the development of novel therapies and the discovery of potential biomarkers. The ability to generate functional endothelial cells (ECs) from induced pluripotent stem cells (iPSCs) from small amounts of blood is a novel and powerful tool for cell-based therapies. Human iPSC-derived ECs (iPS-ECs) have a broad range of clinical applications including cell-based therapy, disease modelling and drug screening; they can be used in mechanistic studies towards the development of novel therapies and in the discovery of new biomarkers to be applied in regenerative medicine and treatment of diabetic vasculopathy. Here we utilize transcriptomic and proteomic technologies to assess patient-specific iPS-ECs from diabetic (DiPS-ECs) and non-diabetic (NiPS-ECS) donors $1,2,3,4$ in order to investigate the mechanisms driving endotheliopathy in diabetes. Our in vitro and in vivo models recapitulate the effects of hyperglycaemia on the vasculature in the clinical setting. RNA-seq data showed that genes and proteins involved in angiogenesis and EC function were significantly downregulated in DiPS-ECs in comparison to NiPS-ECS $(n=3, p<0.05)$. Specific epsins regulating VEGF-mediated angiogenesis were downregulated in DiPS-ECs, leading to increased signalling VEGF pathway activation6. Moreover factors involved in E-cadherin signalling, endothelial-to-mesenchymal transition and fibrosis were increased in DiPS-ECs. We detected abnormal capillary permeability and barrier integrity in DiPS-ECs using xCELLigence ${ }^{\circledR}$. DiPS-ECs had significantly reduced barrier integrity and barrier recovery $(n=3, p<0.001, \pm S E M)$ and also displayed impaired tube formation in vitro $(\mathrm{n}=3, \pm$ SEM, $\mathrm{p}<0.05)$. DiPS-ECs displayed impaired function demonstrated by decreased blood flow recovery (BFR) compared to NiPS ECs $(n=3)$ when injected to the hindlimb of mice following femoral artery ligation. Finally, our proteomic and transcriptomic analysis confirmed imbalances in several angiogenic genes including endothelial specific Roundabout protein 4 (ROBO4) that is highly involved in pathways related to angiogenesis, barrier stability and endothelial health 7. Expression of ROBO4 was found to be impaired in DiPS-ECs and transcriptomic analysis along with in vitro and in vivo studies revealed its importance in vascular development and angiogenesis. Our data support the impaired angiogenic functionality of DiPSECs cells in vitro and in vivo and show that DiPS-ECs carry an imprint of the diabetic milieu which is reflected in their dysfunction. To the best of our knowledge, we have identified a novel disease-specific signature in diabetic iPS-ECs, therefore our human iPS-EC model may serve as a valuable tool to study biological pathways and identify new treatments for diabetes-induced endotheliopathy.

Conflict of Interest None

\section{PRIMARY MITRAL REGURGITATION SUCCESSFULLY TREATED BY PERCUTANEOUS MITRAL VALVE LEAFLET REPAIR RESULTS IN POSITIVE CARDIAC REVERSE REMODELLING AND FUNCTIONAL IMPROVEMENT}

${ }^{1}$ Thomas Craven, ${ }^{1}$ Pei Gee Chew, 'Miroslawa Gorecka, 'Louise Brown, 'Arka Das, ${ }^{1}$ Amrit Chowdhary, ${ }^{1}$ Nicholas Jex, ${ }^{1}$ Sharmaine Thirunavukarasu, 'Erica Dall'Armellina, ${ }^{2}$ Eylem Levelt, ${ }^{3}$ Dominik Schlosshan, ${ }^{3}$ Christopher Malkin, ${ }^{3}$ Daniel Blackman, 'Sven Plein, ${ }^{1}$ John Greenwood. 'University of Leeds, Leeds, UK; ${ }^{2}$ University of Leeds, Multidisciplinary Cardiovascular Research Centre and Biomedical Imaging Science; ${ }^{3}$ Leeds Teaching Hospitals NHS trust

\subsection{6/heartjin-2021-BCS.10}

Background Percutaneous mitral valve leaflet repair can be an effective treatment for primary mitral regurgitation (MR) patients deemed high-risk for surgery. Accurate assessment of cardiac reverse remodelling is essential to optimise future patient selection. Cardiovascular magnetic resonance (CMR) is the reference standard for cardiac volumetric assessment and compared to transthoracic echocardiography (TTE) provides superior reproducibility in MR quantification. Prior CMR studies have analysed cardiac reverse remodelling following percutaneous intervention in combined cohorts of primary and secondary MR patients. However, as aetiology of MR can significantly impact outcomes, focused studies are warranted. Therefore, we aimed to assess cardiac reverse remodelling and quantify changes in MR following percutaneous mitral valve leaflet repair for primary MR using the reference standard (CMR).

Methods 12 patients with at least moderate-severe MR on TTE were prospectively recruited to undergo CMR imaging and 6-minute walk tests (6MWT) at baseline and 6 months following percutaneous mitral valve leaflet repair (MitraClip). CMR protocol involved: left-ventricular (LV) short axis cines (bSSFP, SENSE-2, 10mm, no gap), transaxial right-ventricular (RV) cines (bSSFP, SENSE-2, $8 \mathrm{~mm}$, no gap), two and four chamber cines and aortic through-plane phase contrast imaging, planned at the sino-tubular junction. MR was quantified indirectly using LV and aortic stroke volumes.

Results 12 patients underwent percutaneous mitral valve leaflet repair (MitraClip) for posterior mitral valve leaflet prolapse, however 1 patient declined follow up after single-leaflet clip 